TRANSACTIONS OF THE

AMERICAN MATHEMATICAL SOCIETY

Volume 357, Number 9, Pages 3739-3755

S 0002-9947(05)03692-5

Article electronically published on March 31, 2005

\title{
APPLICATIONS OF THE WOLD DECOMPOSITION TO THE STUDY OF ROW CONTRACTIONS ASSOCIATED WITH DIRECTED GRAPHS
}

\author{
ELIAS KATSOULIS AND DAVID W. KRIBS
}

\begin{abstract}
Based on a Wold decomposition for families of partial isometries and projections of Cuntz-Krieger-Toeplitz-type, we extend several fundamental theorems from the case of single vertex graphs to the general case of countable directed graphs with no sinks. We prove a Szego-type factorization theorem for CKT families, which leads to information on the structure of the unit ball in free semigroupoid algebras, and show that joint similarity implies joint unitary equivalence for such families. For each graph we prove a generalization of von Neumann's inequality which applies to row contractions of operators on Hilbert space which are related to the graph in a natural way. This yields a functional calculus determined by quiver algebras and free semigroupoid algebras. We establish a generalization of Coburn's theorem for the $\mathrm{C}^{*}$-algebra of a CKT family, and prove a universality theorem for $\mathrm{C}^{*}$-algebras generated by these families. In both cases, the $\mathrm{C}^{*}$-algebras generated by quiver algebras play the universal role.
\end{abstract}

\section{INTRODUCTION}

In 20], the second author and Jury derived a Wold decomposition for families of partial isometries which satisfy the "Cuntz-Krieger-Toeplitz" directed graph relations, which we call the $(\dagger)$ relations. This theorem may also be obtained as a special case of the Wold decomposition recently established by Muhly and Solel 30. for the more general setting of induced representations of tensor algebras. In 20] it was used to investigate the internal structure of free semigroupoid algebras [18, 20, 22, 26, 27] and a dilation theory was built around it in [19, 31. In this paper, we apply this Wold decomposition to establish extensions of a number of fundamental theorems to the case of general countable directed graphs with no sinks, whereby the extended results can be regarded as occurring for single vertex graphs.

The next section ( $\S 2)$ contains background information. In $\S 3$ we include an expanded discussion of the Wold decomposition and extend a folklore result for isometries on Hilbert space: We prove that CKT families $\left\{S_{e}, P_{x}\right\}$ of partial isometries and projections are jointly similar precisely when they are jointly unitarily equivalent. We also establish a Szego-type factorization theorem [37, 35] for CKT

Received by the editors November 11, 2003 and, in revised form, March 15, 2004.

2000 Mathematics Subject Classification. Primary 47A63, 47L40, 47L80.

Key words and phrases. Directed graph, partial isometry, row contraction, Wold decomposition, von Neumann inequality, Cuntz-Krieger $\mathrm{C}^{*}$-algebra, quiver algebra, free semigroupoid algebra. 
families. In $\S 4$ we use the Szego theorem to obtain detailed information [11] on the unit ball of semigroupoid algebras which are partly free [26, 27].

Given a countable directed graph $G$ with no sinks, in $\S 5$ we show there is a von Neumann inequality for the set of row contractions $T=\left(T_{1}, \ldots, T_{n}\right)$ of operators on Hilbert space such that the joint actions of the $T_{i}$ are related to $G$ in a natural way. In the case of single vertex graphs, we recover the original form of the von Neumann inequality [42, 39] (when $n=1$ ) and Popescu's version [34] (when $2 \leq n \leq \infty$ ). If $n \geq 2$, the estimates obtained are sharper than the estimates of [34 in general. In fact, we show these estimates respect the partial ordering on directed graphs induced by graph deformations. An immediate consequence of the von Neumann inequality is the existence of functional calculus $(\S 6)$ for row contractions based on quiver algebras $22,26,27,29,30,31$ and free semigroupoid algebras. There is a long history of both positive and negative results associated with von Neumann inequalities for the multivariable setting, and this work fits in with such efforts. See 1, 2, 3, 6, 9, 12, 17, 31, 34] for some of the different perspectives. In connection with the current work, see 31] where a more abstract form of the von Neumann inequality is obtained.

In the final section, we apply the Wold decomposition to obtain a pair of universality theorems for directed graph $\mathrm{C}^{*}$-algebras. In particular, a generalization of Coburn's theorem [8, 33 for isometries on Hilbert space is established: The $\mathrm{C}^{*}$-algebra generated by any CKT family which satisfies a natural non-unitary condition is isomorphic to the $\mathrm{C}^{*}$-algebra generated by the corresponding quiver algebra. Further, we prove that the $\mathrm{C}^{*}$-algebras generated by quiver algebras are the universal $\mathrm{C}^{*}$-algebras for the CKT relations.

\section{Preliminaries}

Let $G$ be a countable directed graph with directed edges $\mathcal{E}(G)$ and vertices $\mathcal{V}(G)$. Given a finite path $w$ in $G$, we write $w=y w x$ when the source vertex of $w$ is $s(w)=x$ and the range vertex of $w$ is $r(w)=y$. (For vertices $x \in \mathcal{V}(G)$ put $r(x)=x=s(x)$.) Say that $G$ has a source at $x \in \mathcal{V}(G)$ if there are no edges which finish at $x$; that is, $r(e) \neq x$ for $e \in \mathcal{E}(G)$. Similarly, $G$ has a sink at $x \in \mathcal{V}(G)$ if $s(e) \neq x$ for $e \in \mathcal{E}(G)$. Define the free semigroupoid $\mathbb{F}^{+}(G)$ to be the set of all vertices $\mathcal{V}(G)$ and all finite paths in $G$, written in reduced form, with the natural operations of concatenation of paths allowed by the graph structure. A path $w=e_{k} \cdots e_{1} \in \mathbb{F}^{+}(G)$ is said to be a loop if $s(w)=r(w)$. If in addition, $r\left(e_{i}\right) \neq r\left(e_{j}\right)$, for $i \neq j$, then $w$ is said to be vertex-simple. A vertex-simple loop $w=e_{k} \cdots e_{1}, e_{i} \in \mathcal{E}(G)$, has an entrance if there exists an edge $f \in \mathcal{E}(G)$ such that $r(f)=r\left(e_{i}\right)$, for some $i$, but $f \neq e_{i}$. Finally, let $\mathcal{P}_{G}^{+}$be the path algebra generated by $G$; this is the set of polynomials with complex coefficients and monomials belonging to $\mathbb{F}^{+}(G)$.

Given a family of nonzero partial isometries $\left\{S_{e}: e \in \mathcal{E}(G)\right\}$ and projections $\left\{P_{x}: x \in \mathcal{V}(G)\right\}$ which act on a common Hilbert space, consider the following relations:

$$
(\dagger)\left\{\begin{array}{lll}
(i) & P_{x} P_{y}=0 & \text { for all } x, y \in \mathcal{V}(G), x \neq y \\
(\text { ii }) & S_{e}^{*} S_{f}=0 & \text { for all } e, f \in \mathcal{E}(G), e \neq f \\
(\text { iii }) & S_{e}^{*} S_{e}=P_{s(e)} & \text { for all } e \in \mathcal{E}(G) \\
(\text { iv }) & \sum_{r(e)=x} S_{e} S_{e}^{*} \leq P_{x} & \text { for all } x \in \mathcal{V}(G)
\end{array}\right.
$$


If equality is achieved in $(i v)$ for all $x \in \mathcal{V}(G)$, then we refer to the relations as ( $\ddagger)$. Observe that $(\ddagger)$ can only occur for $G$ with no sources. We mention that the operator algebras generated by families $\left\{S_{e}, P_{x}\right\}$ which satisfy $(\dagger)$ have been the focus of intense recent interest. They include so-called Cuntz-Krieger directed graph $\mathrm{C}^{*}$-algebras, free semigroupoid algebras, quiver algebras, etc. (The references $[4,10,18,20,22,23,24,25,26,27,29,30,31,38,40]$ give a starting point for the interested reader.) Let us mention at this point the existence of the universal $\mathrm{C}^{*}$-algebra $\mathrm{C}^{*}(G)[23,24,25$ for the relations $(\ddagger)$ for a given directed graph $G$. From these operator algebra motivations, we refer to $(\ddagger)$ as the Cuntz-Krieger (CK) relations, and $(\dagger)$ as the Cuntz-Krieger-Toeplitz relations (CKT).

We next describe the prototypical "pure model" [19, 20] in the Wold decomposition used here. (More details are given in the next section.) Let $G$ be a countable directed graph (with no sinks) and define $\mathcal{H}_{G}$ to be the Hilbert space with orthonormal basis given by $\left\{\xi_{w}: w \in \mathbb{F}^{+}(G)\right\}$. This generalized "Fock space" associated with a directed graph was introduced by Muhly [29]. For $e \in \mathcal{E}(G)$ define partial isometries $L_{e}$ on $\mathcal{H}_{G}$ by

$$
L_{e} \xi_{w}=\left\{\begin{array}{cl}
\xi_{e w} & \text { if } r(w)=s(e) \\
0 & \text { otherwise }
\end{array}\right.
$$

Observe that $L_{e}^{*} L_{e}=P_{s(e)}$ is the projection onto $\operatorname{span}\left\{\xi_{w}: r(w)=s(e)\right\}$, and that the family $\left\{L_{e}, P_{x}\right\}$ satisfies $(\dagger)$. For $x \in \mathcal{V}(G)$, we define the "tree component" subspace $\mathcal{H}_{x}=\operatorname{span}\left\{\xi_{w}: w=w x\right\}$. Notice that these subspaces are reducing for the $L_{e}$. In fact, the subspaces $\left\{\mathcal{H}_{x}: x \in \mathcal{V}(G)\right\}$ form the unique family of minimal nonzero joint reducing subspaces for the $L_{e}$ [26].

Given a directed graph $G$, the associated quiver algebra $\mathcal{A}_{G}$ is the norm-closed algebra generated by $\left\{L_{e}, P_{x}: e \in \mathcal{E}(G), x \in \mathcal{V}(G)\right\}$, where the $P_{x}$ here are the vertex projections associated with the pure model (1). The associated free semigroupoid algebra $\mathfrak{L}_{G}$ is the weak operator topology closure of $\mathcal{A}_{G}$. The commutant of $\mathfrak{L}_{G}$ coincides with $\mathfrak{L}_{G}^{\prime}=\mathfrak{R}_{G}$ [26], the wOT-closed algebra generated by partial isometries $R_{e}$, defined on $\mathcal{H}_{G}$ (as in (1)) by $R_{e} \xi_{w}=\xi_{\text {we }}$, together with their initial projections which we denote by $Q_{x}, x \in \mathcal{V}(G)$. The projections $Q_{x}$ are precisely the projections onto the tree component subspaces $\mathcal{H}_{x}$.

We mention that the graph $G$ was proved to be a complete invariant for $\mathcal{A}_{G}$ (and $\mathfrak{L}_{G}$ ) up to unitary equivalence by Kribs and Power [26]. More recently, Solel 38] proved $G$ is an invariant for isometric isomorphism and, independently, the authors [22] proved that $G$ is a banach algebra isomorphism invariant for $\mathcal{A}_{G}$. (In fact, when $G$ has no sources or no sinks it was proved in 22] that $G$ is an algebraic isomorphism invariant for $\mathcal{A}_{G}$.) This is a true departure from the $\mathrm{C}^{*}$-algebra case, where, for instance, it is not hard to see that $G$ is not a $*$-isomorphism invariant for $\mathrm{C}^{*}(G)$. (For example, for many non-isomorphic graphs, $\mathrm{C}^{*}(G)$ is $*$-isomorphic to the set of compact operators.)

\section{WOLD DECOMPOSITION}

The Wold decomposition from [20] (which assumed $G$ had no sinks) asserts that every set of partial isometries $\left\{S_{e}: e \in \mathcal{E}(G)\right\}$ which satisfies (†) on a Hilbert space $\mathcal{H}$ is jointly unitarily equivalent to a direct sum

$$
S_{e} \simeq V_{e} \oplus\left(\left.\sum_{x \in V(G)} \oplus L_{e}^{\left(\alpha_{x}\right)}\right|_{\mathcal{H}_{x}^{\left(\alpha_{x}\right)}}\right) \quad \text { for } \quad e \in E(G)
$$


where $V=\left(V_{e}\right)_{e \in \mathcal{E}(G)}$ satisfies $(\ddagger)$, and hence determines a representation of a Cuntz-Krieger directed graph $\mathrm{C}^{*}$-algebra. As indicated above, this result can be derived from a special case of the Wold decomposition from [30]. The restricted ampliations of the $L_{e}$ in this decomposition are said to form the pure part of the dilation. (Notice that the pure model (1) is captured with $V_{e} \equiv 0$ and $\alpha_{x} \equiv 1$.) The $\alpha_{x}$ are called the vertex multiplicities in the dilation and are computed from $\left\{S_{e}, P_{x}\right\}$ via the equations

$$
\alpha_{x}=\operatorname{rank}\left(P_{x}\left(I-\sum_{e} S_{e} S_{e}^{*}\right)\right) \quad \text { for } \quad x \in \mathcal{V}(G) .
$$

More specifically, the joint unitary equivalence (2) arises from the spatial decomposition $\mathcal{H}=\mathcal{H}_{c} \oplus \mathcal{H}_{p}$ where $\mathcal{H}_{c}=\mathcal{H}_{p}^{\perp}$ and $\mathcal{H}_{p}=\sum_{w \in \mathbb{F}^{+}(G)} \oplus w(S) \mathcal{W}$ are joint reducing subspaces for the $S_{e}$, with $\mathcal{W}$ the wandering subspace for $S$ given by

$$
\mathcal{W}=\operatorname{Ran}\left(I-\sum_{e} S_{e} S_{e}^{*}\right)=\bigcap_{e} \operatorname{ker} S_{e}^{*} .
$$

Observe for $x \in \mathcal{V}(G)$ that $P_{x} \mathcal{W}=\operatorname{Ran}\left(P_{x}-\sum_{r(e)=x} S_{e} S_{e}^{*}\right)$ is a subspace of $\mathcal{W}$. The subspaces $\mathcal{H}_{c}$ and $\mathcal{H}_{p}$ have the alternate descriptions

$$
\begin{aligned}
\mathcal{H}_{p} & =\left\{\xi \in \mathcal{H}: \lim _{d \rightarrow \infty} \sum_{w \in \mathbb{F}^{+}(G)_{d}}\left\|w(S)^{*} \xi\right\|^{2}=0\right\}, \\
\mathcal{H}_{c} & =\bigcap_{d \geq 1}\left\{\xi \in \mathcal{H}: \sum_{w \in \mathbb{F}^{+}(G)_{d}} w(S) w(S)^{*} \xi=\xi\right\},
\end{aligned}
$$

where $\mathbb{F}^{+}(G)_{d}$ is the set of paths inside $\mathbb{F}^{+}(G)$ of length $d$.

As a direct consequence of the Wold decomposition, we prove a generalization of the well-known fact that similar isometries are unitarily equivalent. This result also generalizes Popescu's theorem [33] for the case of $n$-tuples of isometries with orthogonal ranges, which occurs below when $G$ is a single vertex graph with $n \geq 2$ edges.

Theorem 3.1. Let $G$ be a countable directed graph with no sinks. If $\left\{S_{e}, P_{x}\right\}$ and $\left\{S_{e}^{\prime}, P_{x}^{\prime}\right\}$ both satisfy $(\dagger)$ for $G$ and are jointly similar, then they are jointly unitarily equivalent. That is, if there is an invertible operator $A$ with

$$
\begin{aligned}
& A S_{e} A^{-1}=S_{e}^{\prime} \quad \text { for } \quad e \in \mathcal{E}(G), \\
& A P_{x} A^{-1}=P_{x}^{\prime} \quad \text { for } \quad x \in \mathcal{V}(G),
\end{aligned}
$$

then there is a unitary operator $U$ such that

$$
\begin{aligned}
U S_{e} U^{*}=S_{e}^{\prime} & & \text { for } & e \in \mathcal{E}(G), \\
U P_{x} U^{*}=P_{x}^{\prime} & & \text { for } & x \in \mathcal{V}(G) .
\end{aligned}
$$

Proof. Without loss of generality, assume both families of operators act on $\mathcal{H}$. Let $\mathcal{H}=\mathcal{H}_{c} \oplus \mathcal{H}_{p}=\mathcal{H}_{c}^{\prime} \oplus \mathcal{H}_{p}^{\prime}$ be the spatial decompositions associated with the Wold decompositions (2) for the $S_{e}$ and $S_{e}^{\prime}$ respectively.

For $e \in \mathcal{E}(G)$ let

$$
\left.S_{e}\right|_{\mathcal{H}_{c}} \equiv V_{e},\left.\quad S_{e}\right|_{\mathcal{H}_{p}} \equiv W_{e},\left.\quad S_{e}^{\prime}\right|_{\mathcal{H}_{c}^{\prime}} \equiv V_{e}^{\prime},\left.\quad S_{e}^{\prime}\right|_{\mathcal{H}_{p}^{\prime}} \equiv W_{e}^{\prime}
$$

By hypothesis, for $e \in \mathcal{E}(G)$ we have

$$
\operatorname{ker} S_{e}^{*}=\operatorname{ker}\left(A^{*} S_{e}^{* *}\left(A^{*}\right)^{-1}\right)=A^{*}\left(\operatorname{ker}\left(S_{e}^{* *}\right)\right) .
$$


Let $\mathcal{W}$ and $\mathcal{W}^{\prime}$ be the wandering subspaces for $S$ and $S^{\prime}$ respectively. Then

$$
A^{*}\left(\mathcal{W}^{\prime}\right)=A^{*}\left(\bigcap_{e} \operatorname{ker} S_{e}^{*}\right)=\bigcap_{e} \operatorname{ker} S_{e}^{*}=\mathcal{W} .
$$

In particular, $\operatorname{dim} \mathcal{W}=\operatorname{dim} \mathcal{W}^{\prime}$. But more is true. Recall that the vertex multiplicities in the pure parts of the Wold decompositions for $S$ and $S^{\prime}$ are given by $\alpha_{x}=\operatorname{dim} P_{x} \mathcal{W}$ and $\alpha_{x}^{\prime}=\operatorname{dim} P_{x} \mathcal{W}^{\prime}$. By hypothesis and the previous identity we have

$$
P_{x} \mathcal{W}=P_{x} A^{*} \mathcal{W}^{\prime}=A^{*} P_{x}^{\prime} \mathcal{W}^{\prime} \quad \text { for } \quad x \in \mathcal{V}(G),
$$

and hence the invertibility of $A$ yields $\alpha_{x}=\alpha_{x}^{\prime}$ for $x \in \mathcal{V}(G)$.

Thus, it is easily seen from the Wold decomposition that there is a unitary $U_{1}: \mathcal{H}_{p} \rightarrow \mathcal{H}_{p}^{\prime}$ which intertwines the pure parts of $S$ and $S^{\prime}$

$$
U_{1} W_{e} U_{1}^{*}=W_{e}^{\prime} \quad \text { for } \quad e \in \mathcal{E}(G)
$$

Since $G$ has no sinks, every vertex projection $P_{x}$ is the initial projection for some $S_{e}$. Further, recall that $\mathcal{H}_{p}$ (respectively $\mathcal{H}_{p}^{\prime}$ ) is a reducing subspace for the $S_{e}$ $\left(\right.$ respectively $S_{e}^{\prime}$ ). It follows that the restricted vertex projections $\left.P_{x}\right|_{\mathcal{H}_{p}}$ and $\left.P_{x}^{\prime}\right|_{\mathcal{H}_{p}^{\prime}}$ are also intertwined by $U_{1}$.

We now show that the restrictions to $\mathcal{H}_{c}, \mathcal{H}_{c}^{\prime}$ may be spatially intertwined as well. As $A S_{e}=S_{e}^{\prime} A$ and $S_{e} A^{-1}=A^{-1} S_{e}^{\prime}$ for $e \in \mathcal{E}(G)$, equation (4) applied for the $S_{e}$ and the $S_{e}^{\prime}$ implies that $A \mathcal{H}_{c}=\mathcal{H}_{c}^{\prime}$. Let $A_{0}=\left.A\right|_{\mathcal{H}_{c}}: \mathcal{H}_{c} \rightarrow \mathcal{H}_{c}^{\prime}$ and observe that $A_{0}$ is invertible. Then we have

$$
A_{0} V_{e}=V_{e}^{\prime} A_{0} \quad \text { for } \quad e \in \mathcal{E}(G) .
$$

In particular, equation (4) gives

$$
A_{0}=A_{0}\left(I_{\mathcal{H}_{c}}\right)=A_{0}\left(\sum_{e} V_{e} V_{e}^{*}\right)=\sum_{e} V_{e}^{\prime} A_{0} V_{e}^{*}
$$

Thus the $(\dagger)$ relations and the hypothesis implies that for $e \in \mathcal{E}(G)$,

$$
V_{e}^{\prime *} A_{0}=V_{e}^{\prime *} V_{e}^{\prime} A_{0} V_{e}^{*}=P_{s(e)}^{\prime} A_{0} V_{e}^{*}=A_{0} P_{s(e)} V_{e}^{*}=A_{0} V_{e}^{*} .
$$

It follows from the adjoint of this identity that

$$
V_{e} A_{0}^{*} A_{0}=A_{0}^{*} V_{e}^{\prime} A_{0}=A_{0}^{*} A_{0} V_{e} \quad \text { for } \quad e \in \mathcal{E}(G) .
$$

Consider the polar decomposition of $A_{0}=U_{2} B$, where $U_{2}: \mathcal{H}_{c} \rightarrow \mathcal{H}_{c}^{\prime}$ is unitary and $B=\left(A_{0}^{*} A_{0}\right)^{1 / 2}$ is an invertible operator on $\mathcal{H}_{c}$. By (6) we have

$$
V_{e} B=B V_{e} \text { for } \quad e \in \mathcal{E}(G),
$$

and it follows that

$$
\begin{aligned}
V_{e}^{\prime} U_{2}=V_{e}^{\prime} U_{2} B B^{-1} & =V_{e}^{\prime} A_{0} B^{-1} \\
& =A_{0} V_{e} B^{-1}=U_{2} B V_{e} B^{-1}=U_{2} V_{e} .
\end{aligned}
$$

Thus

$$
U_{2} V_{e}=V_{e}^{\prime} U_{2} \quad \text { for } \quad e \in \mathcal{E}(G) .
$$

Therefore, we may define a unitary $U=U_{1} \oplus U_{2}$ on $\mathcal{H}$ so that (5) and (7) imply

$$
U S_{e}=S_{e}^{\prime} U^{*} \text { for } \quad e \in \mathcal{E}(G) .
$$

As $G$ has no sinks, it follows that the projections $P_{x}$ and $P_{x}^{\prime}$ are also intertwined by the unitary $U$. 
We now present another application of the Wold decomposition that allows us to prove a Szego-type factorization theorem. This theorem generalizes earlier results of Rosenblum and Rovnyak [37, Theorem 3.4] and Popescu [35].

Theorem 3.2. Let $G$ be a countable directed graph with no sinks and let $\left\{P_{x}\right\}_{x \in \mathcal{V}(G)}$ and $\left\{S_{e}\right\}_{e \in \mathcal{E}(G)}$ be sets of projections and partial isometries which satisfy $(\dagger)$. Assume that $\left\{S_{e}\right\}_{e \in \mathcal{E}(G)}$ is pure. If $Y \in B(\mathcal{H})$ is a positive invertible operator, then the following assertions are equivalent:

(i) $Y=A^{*} A$ for some $A \in B(\mathcal{H})$ which commutes with $\left\{P_{x}\right\}_{x \in \mathcal{V}(G)}$ and $\left\{S_{e}\right\}_{e \in \mathcal{E}(G)}$.

(ii) $S_{e}^{*} Y S_{f}=Y S_{e}^{*} S_{f}=S_{e}^{*} S_{f} Y$ for all $e, f \in \mathcal{E}(G)$.

Proof. We need to prove that (ii) implies (i). Let $T_{u} \equiv Y^{1 / 2} S_{u} Y^{-1 / 2}$ for $u \in \mathbb{F}^{+}(G)$. We will show that the family $\left\{T_{e}\right\}_{e \in \mathcal{E}(G)}$ is jointly unitarily equivalent to $\left\{S_{e}\right\}_{e \in \mathcal{E}(G)}$. Towards this end notice that $T_{e}^{*} T_{e}=P_{s(e)}$ and $T_{e} T_{e}^{*} \leq P_{r(e)}$. Indeed,

$$
\begin{aligned}
T_{e}^{*} T_{e} & =Y^{-1 / 2} S_{e}^{*} Y S_{e} Y^{-1 / 2} \\
& =Y^{-1 / 2} Y Y^{-1 / 2} P_{s(e)}=P_{s(e)}=S_{e}^{*} S_{e},
\end{aligned}
$$

and a similar calculation shows the claimed inequality. Therefore, the collections $\left\{P_{x}\right\}_{x \in \mathcal{V}(G)}$ and $\left\{T_{e}\right\}_{e \in \mathcal{E}(G)}$ satisfy the requirements of the Wold Decomposition; i.e., the relations $(\dagger)$, and therefore the earlier discussion applies. First, we determine that the Cuntz-Krieger part of $\left\{T_{e}\right\}_{e \in \mathcal{E}(G)}$ is trivial. Indeed,

$$
\begin{aligned}
\mathcal{H}_{c} & =\bigwedge_{k \in \mathbb{N}} \bigvee_{|u|=k} T_{u}\left(\mathcal{H}_{G}\right) \\
& =\bigwedge_{k \in \mathbb{N}} \bigvee_{|u|=k} Y^{1 / 2} S_{u}(\mathcal{H}) \\
& =Y^{1 / 2}\left(\bigwedge_{k \in \mathbb{N}} \bigvee_{|u|=k} S_{u}(\mathcal{H})\right)=\{0\} .
\end{aligned}
$$

Thus $\left\{T_{e}\right\}_{e \in \mathcal{E}(G)}$ is pure. Let $\widetilde{\mathcal{W}}$ be its wandering subspace and let $\widetilde{\mathcal{W}}_{x} \equiv P_{x} \widetilde{\mathcal{W}} \subseteq$ $\widetilde{\mathcal{W}}$. Then by the Wold decomposition there exists a unitary operator

$$
V: \mathcal{H} \longrightarrow \sum_{x \in \mathcal{V}(G)} \oplus\left(Q_{x} \mathcal{H}_{G}\right)^{\left(\beta_{x}\right)}
$$

such that for any $e \in \mathcal{E}(G)$,

$$
V T_{e} V^{*}=\left.\sum_{x \in \mathcal{V}(G)} \oplus L_{e}^{\left(\beta_{x}\right)}\right|_{\left(Q_{x} \mathcal{H}_{G}\right)^{\left(\beta_{x}\right)}},
$$

where $\beta_{x}=\operatorname{dim} \widetilde{\mathcal{W}}_{x}, x \in \mathcal{V}(G)$. Similarly, there exists a unitary

$$
U: \mathcal{H} \longrightarrow \sum_{x \in \mathcal{V}(G)} \oplus\left(Q_{x} \mathcal{H}_{G}\right)^{\left(\alpha_{x}\right)}
$$

such that for any $e \in \mathcal{E}(G)$,

$$
U S_{e} U^{*}=\left.\sum_{x \in \mathcal{V}(G)} \oplus L_{e}^{\left(\alpha_{x}\right)}\right|_{\left(Q_{x} \mathcal{H}_{G}\right)^{\left(\alpha_{x}\right)}}
$$

In order to establish the joint unitary equivalence between the families $\left\{T_{e}\right\}_{e \in \mathcal{E}(G)}$ and $\left\{S_{e}\right\}_{e \in \mathcal{E}(G)}$, we will prove that $V T_{e} V^{*}=U^{*} S_{e} U, e \in \mathcal{E}(G)$. From what we 
have proved so far, it follows that we only need to verify $\beta_{x}=\alpha_{x}$, for all $x \in \mathcal{V}(G)$. Fix an $x \in \mathcal{V}(G)$ and let $\xi \in \widetilde{\mathcal{W}}_{x}$. Then for all $e \in \mathcal{E}(G)$ and $\zeta \in \mathcal{H}_{G}$, we have

$$
\left(Y^{1 / 2} \xi, S_{e} \zeta\right)=\left(\xi, Y^{1 / 2} S_{e} \zeta\right)=\left(\xi, T_{e} Y^{1 / 2} \zeta\right)=0
$$

Thus by the invertibility of $Y$ we have $Y^{1 / 2} \widetilde{\mathcal{W}}=\mathcal{W}$. But $Y^{1 / 2} T_{e}^{*} T_{e}=S_{e}^{*} S_{e} Y^{1 / 2}$ for $e \in \mathcal{E}(G)$ since the same is true for $Y$. Hence, as $G$ has no sinks, every $P_{x}$ is equal to some initial projection $S_{e}^{*} S_{e}=T_{e}^{*} T_{e}$ and it follows that $Y^{1 / 2} \widetilde{\mathcal{W}}_{x}=\mathcal{W}_{x}$ for $x \in \mathcal{V}(G)$ since $Y^{1 / 2}$ is invertible. Therefore, the identity $\beta_{x}=\alpha_{x}$ holds for all $x \in \mathcal{V}(G)$, as required.

We have shown that $V T_{e} V^{*}=U S_{e} U^{*}$ for $e \in \mathcal{E}(G)$. In particular, this implies that $U^{*} V Y^{1 / 2} S_{e}=S_{e} U^{*} V Y^{1 / 2}$, for all $e \in \mathcal{E}(G)$, and the operator $U^{*} V Y^{1 / 2}$ commutes with $\left\{S_{e}\right\}_{e \in \mathcal{E}(G)}$. Moreover, notice that for $e \in \mathcal{E}(G)$,

$$
\begin{aligned}
S_{e}^{*} S_{e} U^{*} V Y^{1 / 2} & =S_{e}^{*} U^{*} V T_{e} V^{*} V Y^{1 / 2} \\
& =U^{*} V T_{e}^{*} V^{*} V T_{e} Y^{1 / 2}=U^{*} V Y^{1 / 2} T_{e}^{*} T_{e},
\end{aligned}
$$

and it follows that $U^{*} V Y^{1 / 2}$ commutes with $\left\{P_{x}\right\}_{x \in \mathcal{V}(G)}$ as well. The desired operator for $(i)$ is $A=U^{*} V Y^{1 / 2}$.

Using the fact that $\mathfrak{R}_{G}^{\prime}=\mathfrak{L}_{G}$ we obtain the following.

Corollary 3.3. Let $G$ be a countable directed graph with no sinks. If $Y \in B(\mathcal{H})$ is a positive invertible operator, then the following assertions are equivalent:

(i) $Y=A^{*} A$ for some $A \in \mathfrak{L}_{G}$.

(ii) $R_{e}^{*} Y R_{f}=Y R_{e}^{*} R_{f}=R_{e}^{*} R_{f} Y$ for all $e, f \in \mathcal{E}(G)$.

\section{An application to the GeOMEtry of the Unit Ball}

In [1], Davidson, Pitts and the first author proved that every operator in the open unit ball of a $\mathrm{w}^{*}$-closed operator algebra generated by $n$ isometries with orthogonal ranges, is the average of isometries. This result applies in particular to the noncommutative Toeplitz algebra $\mathfrak{L}_{n}$, thus extending a classical result of Marshall 28 from function theory to the noncommutative setting. Using the Szego-type factorization theorem we proved earlier, we extend the result of [11] to a broad class of free semigroupoid algebras.

The techniques of 11] yield a more general result than the one quoted in the introduction. In order to state it, we need the following.

Definition 4.1. An operator algebra $\mathcal{A}$ satisfies the factorization property if for any $A \in \mathcal{A}$ with $\|A\|<1$, the equation $X^{*} X=I-A^{*} A$ has a solution in $X \in \mathcal{A}$.

It is clear from Corollary 3.3 that any free semigroupoid algebra for a graph with no sinks satisfies the factorization property.

Theorem 4.2 ([11). Let $\mathcal{A}$ be a norm closed operator algebra satisfying the following two properties:

(i) $\mathcal{A}$ satisfies the factorization property.

(ii) $\mathcal{A}$ contains two isometries with orthogonal ranges.

If $\|A\|<1-\frac{1}{k}, A \in \mathcal{A}, k \in \mathbb{N}$, then $A$ is the average of $6 k$ isometries.

Remark 4.3. The above result is not explicitly stated as a theorem in [11] but its validity is ascertained on [11, page 118]. 
On the other hand, in [27] Power and the second author identified a property for a directed graph $G$ that is equivalent to the existence of two isometries in $\mathfrak{L}_{G}$ with orthogonal ranges.

Definition 4.4. A countable directed graph satisfies the uniform aperiodic path property if the saturation of each vertex $x \in \mathcal{V}(G)$ either contains two distinct loops or an infinite proper path.

Theorem 4.5 ([27]). Let $G$ be a countable directed graph. Then $G$ satisfies the uniform aperiodic path property if and only if $\mathfrak{L}_{G}$ contains a pair of isometries with orthogonal ranges.

In light of Theorem 4.2 and Corollary [3.3, the previous result provides the last step for establishing the main result of this section.

Theorem 4.6. Let $G$ be a countable directed graph which satisfies the uniform aperiodic path property and let $A \in \mathfrak{L}_{G}$. If $\|A\|<1-\frac{1}{k}, k \in \mathbb{N}$, then $A$ is the average of $6 k$ isometries.

Remark 4.7. What about other free semigroupoid algebras? Clearly, $H^{\infty}$ fails the aperiodic path property and yet the convex combinations of the isometries cover the open unit ball. On the other hand, not all free semigroupoid algebras satisfy that property. Indeed, if $G$ is a graph with two vertices $x, y$ and an edge $e=x e y$, then $\mathfrak{L}_{G}$ is the direct sum of the $2 \times 2$ upper triangular matrices with $\mathbb{C}$. The convex hull of the isometries in this algebra consists of the diagonal matrices.

\section{Von NeUmann INEQUALity}

Given a row contraction $T=\left(T_{1}, \ldots, T_{n}\right)$ of (nonzero) operators acting on a Hilbert space $\mathcal{H}$, consider a family of mutually orthogonal projections $\mathcal{P}=\left\{P_{x}\right\}_{x \in \mathcal{J}}$ on $\mathcal{H}$ which sum to the identity operator and stabilize $T$ in the following sense:

$$
T_{i} P_{x}, P_{x} T_{i} \in\left\{T_{i}, 0\right\} \quad \text { for } \quad 1 \leq i \leq n \text { and } \quad x \in \mathcal{J} .
$$

Observe that these relations determine a directed graph $G$ with vertex set $\mathcal{V}(G) \equiv \mathcal{J}$ and $n$ directed edges $e_{1}, \ldots, e_{n}$, where $r\left(e_{i}\right)$ and $s\left(e_{i}\right)$ are the unique vertices with $P_{r\left(e_{i}\right)} T_{i} P_{s\left(e_{i}\right)}=T_{i}$. When there is no confusion, we shall write $T=\left(T_{e}\right)_{e \in \mathcal{E}(G)}$ if an ordering of the $T_{i}$ has been induced by a fixed projection set $\mathcal{P}$.

Let $T=\left(T_{e}\right)_{e \in E(G)}$ and $\mathcal{P}=\left\{P_{x}\right\}_{x \in \mathcal{V}(G)}$ satisfy (8) on $\mathcal{H}$ for a graph $G$ with no sinks. Then it was shown in [19, and previously in a more abstract form 31 for the general setting of tensor algebras over $\mathrm{C}^{*}$-correspondences, that there are partial isometries $\left\{S_{e}: e \in \mathcal{E}(G)\right\}$ on a space $\mathcal{K} \supseteq \mathcal{H}$ such that

(a) $S=\left(S_{e}\right)_{e \in E(G)}$ satisfy $(\dagger)$.

(b) $\mathcal{H}$ reduces each $S_{e}^{*} S_{e}, e \in \mathcal{E}(G)$, and $\left\{\left.S_{e}^{*} S_{e}\right|_{\mathcal{H}}\right\}=\mathcal{P}$.

(c) $\mathcal{H}$ is invariant for each $S_{e}^{*}$ with $\left.S_{e}^{*}\right|_{\mathcal{H}}=T_{e}^{*}, e \in \mathcal{E}(G)$.

(d) $\mathcal{K}=\bigvee_{w \in \mathbb{F}^{+}(G)} w(S) \mathcal{H}$.

Such a minimal partially isometric dilation of $T$ is unique up to a unitary equivalence which fixes $\mathcal{H}$. The vertex multiplicities $\alpha_{x}$ in such a dilation are determined by (31) and, in fact, it was shown in [19] that they may be computed from $T, \mathcal{P}$ by

$$
\alpha_{x}=\operatorname{rank}\left(P_{x}\left(I_{\mathcal{H}}-\sum_{e} T_{e} T_{e}^{*}\right)\right) \quad \text { for } \quad x \in \mathcal{V}(G) .
$$


For a fixed directed graph $G$ we shall define the supremum norm $\|p\|_{\infty}$ of a polynomial $p \in \mathcal{P}_{G}^{+}$by $\|p\|_{\infty} \equiv\left\|p\left(L_{G}\right)\right\|$, where $L_{G}=\left(L_{e}\right)_{e \in E(G)}$ is the canonical pure model (1) for $G$. This definition is in line with other settings, where the norm $\|p\|_{\infty}$ is determined by the pure model in this manner. As the simplest example, recall that the norm of a polynomial $p$ on the unit complex disk satisfies $\|p\|_{\infty}=\left\|p\left(U_{+}\right)\right\|$, where $U_{+}$is the unilateral forward shift [39].

Theorem 5.1. Let $G$ be a countable directed graph with no sinks. Let $T=$ $\left(T_{e}\right)_{e \in \mathcal{E}(G)}$ be a row contraction on a Hilbert space $\mathcal{H}$ which satisfies (8) for $\left\{P_{x}\right.$ : $x \in \mathcal{V}(G)\}$. Then

$$
\|p(T)\| \leq\|p\|_{\infty} \quad \text { for all } p \in \mathcal{P}_{G}^{+} .
$$

Proof. Let $S=\left(S_{e}\right)_{e \in \mathcal{E}(G)}$ be the minimal partially isometric dilation of $T$ associated with the projections $\left\{P_{x}\right\}$. Let $p$ belong to $\mathcal{P}_{G}^{+}$. Then $p(T)=\left.P_{\mathcal{H}} p(V)\right|_{\mathcal{H}}$ by virtue of $(a),(b)$ and $(c)$ above, and hence the Wold decomposition implies that

$$
\|p(T)\| \leq\|p(S)\|=\max \{\|p(V)\|,\|p(W)\|\},
$$

where $V=\left(V_{e}\right)_{e \in \mathcal{E}(G)}$ satisfies $(\ddagger)$ and $W=\left(W_{e}\right)_{e \in \mathcal{E}(G)}$ is pure and satisfies $(\dagger)$.

The decomposition (2) of the pure part $W$ of the minimal dilation $S$ clearly implies that

$$
\|p(W)\| \leq\left\|p\left(L_{G}\right)\right\| \equiv\|p\|_{\infty} .
$$

Thus, we shall finish the proof by showing that $\|p(S)\| \leq\left\|p\left(L_{G}\right)\right\|$ for every row contraction $S=\left(S_{e}\right)_{e \in \mathcal{E}(G)}$ which satisfies $(\ddagger)$ for $G$.

To see this, let $S=\left(S_{e}\right)_{e \in \mathcal{E}(G)}$ satisfy $(\ddagger)$ and let $0<r<1$. Then $r S=$ $\left(r S_{e}\right)_{e \in \mathcal{E}(G)}$ is a pure row contraction, and hence the minimal partially isometric dilation of $r S$ with respect to $\left\{S_{e}^{*} S_{e}: e \in \mathcal{E}(G)\right\}$ is given by the form (2) with $V_{e} \equiv 0$. We claim that the vertex multiplicities for this pure dilation $S^{\prime}=\left(S_{e}^{\prime}\right)_{e \in \mathcal{E}(G)}$ satisfy $\alpha_{x} \geq 1$ for $x \in \mathcal{V}(G)$. Indeed, from (9) these multiplicities are determined by

$$
\alpha_{x}=\operatorname{rank}\left(P_{x}-\sum_{e=x e}\left(r S_{e}\right)\left(r S_{e}\right)^{*}\right) \quad \text { for } \quad x \in \mathcal{V}(G) .
$$

Thus if $\alpha_{x}=0$, then the $(\ddagger)$ relations give

$$
\sum_{e=x e} S_{e} S_{e}^{*}=P_{x}=r^{2} \sum_{e=x e} S_{e} S_{e}^{*},
$$

which is clearly a contradiction since the $P_{x}$ are assumed to be nonzero in the $(\dagger)$ relations.

As $\alpha_{x} \geq 1$ for $x \in \mathcal{V}(G)$, we have

$$
\|p(r S)\| \leq\left\|p\left(S^{\prime}\right)\right\|=\left\|p\left(L_{G}\right)\right\| .
$$

Since $0<r<1$ was arbitrary, it follows that $\|p(S)\| \leq\left\|p\left(L_{G}\right)\right\|$, as required.

Remark 5.2. In the case of single vertex graphs with $n$ edges, the above theorem recovers the classical von Neumann inequality [42, 39] (when $n=1$ ) and Popescu's version [34] (when $2 \leq n \leq \infty$ ). We also mention that the conclusion of Theorem 5.1 can be proved through an application of Theorem 3.10 from 31. However, we note that our direct proof via the Wold decomposition is novel and provides a new perspective on the problem. 
5.1. Partially ordered sets of directed graphs. An alternative perspective on Theorem 5.1 is the following: Given a countable directed graph $G$ with no sinks, the theorem shows there is a corresponding von Neumann inequality with estimate given by (for this discussion) $\|p\|_{G, \infty} \equiv\left\|p\left(L_{G}\right)\right\|$ for $p \in \mathcal{P}_{G}^{+}$, recalling that $L_{G}=\left(L_{e}\right)_{e \in \mathcal{E}(G)}$. On the other hand, every directed graph determines a partially ordered set of directed graphs through its deformations. A directed graph $G_{2}$ is a deformation of $G_{1}$ if $G_{2}$ is obtained from $G_{1}$ by identifying certain vertices in $G_{1}$. The partial order is defined by $G_{1} \leq G_{2}$ if and only if $G_{2}$ is a deformation of $G_{1}$. (This perspective was also discussed in [19] in the context of dilation theory, where it was shown that every row contraction $T$ generates such a partially ordered set of directed graphs through its family of minimal partially isometric dilations.) We observe below that the von Neumann inequality estimates respect the ordering in such a partially ordered set of directed graphs.

Let $C_{n}$ be the directed graph with a single vertex and $n$ directed loop edges. The Sz.-Nagy and Popescu estimates are given by $\left\|p\left(L_{C_{n}}\right)\right\|$, where $p$ is allowed to be any polynomial in $n$ noncommuting variables when $n \geq 2$. Let $G$ be a countable directed graph with $n$ edges. Then $L_{G}=\left(L_{e}\right)_{e \in E(G)}$ is a pure row contraction (in the $n$-tuple sense [36]) and hence its (unique) minimal isometric dilation [7, 15, 36] is given by a multiple of $L_{C_{n}}=\left(L_{1}, \ldots, L_{n}\right)$, where the $L_{i}$ are the creation isometries on the full $n$-variable Fock space. Thus, given $L_{G}=\left(L_{e}\right)_{e \in E(G)}$ and such a polynomial $p$, we shall write $p\left(L_{G}\right)$ for the evaluation of $p$ at the $L_{e}$ which arises from the ordering of the $L_{e}$ induced by the minimal isometric dilation of $L_{G}$.

Corollary 5.3. If $G_{1} \leq G_{2}$ are directed graphs with no sinks and n directed edges, then for every polynomial $p$ in $n$ noncommuting variables

$$
\|p\|_{G_{1}, \infty} \leq\|p\|_{G_{2}, \infty} \leq\|p\|_{C_{n}, \infty}
$$

Proof. The relation $G_{1} \leq G_{2}$ can be seen to induce a $G_{2}$-ordering on $L_{G_{1}}$, where the corresponding projections which stabilize $L_{G_{1}}$ are given by the sums of the initial projections for $L_{e}, e \in \mathcal{E}\left(G_{1}\right)$, which are naturally induced by the deformation. Hence the first inequality is a consequence of Theorem 5.1 The last inequality follows from Popescu's von Neumann inequality since $L_{G_{1}}$ and $L_{G_{2}}$ are both row contractions and $n$-tuples.

It is not hard to see that $\|p\|_{G, \infty}$ gives a sharper estimate than $\left\|p\left(L_{C_{n}}\right)\right\|$ in general when $n \geq 2$. Consider the following simple illustration of this fact.

Example 5.4. Let $T_{1}, T_{2}$ be contractions on a Hilbert space $\mathcal{H}$ and define operators on $\mathcal{H}^{(2)}$ by

$$
V_{1}=\left(\begin{array}{cc}
0 & 0 \\
T_{1} & 0
\end{array}\right) \quad \text { and } \quad V_{2}=\left(\begin{array}{cc}
0 & T_{2} \\
0 & 0
\end{array}\right)
$$

Then $V=\left(V_{1}, V_{2}\right)$ is a row contraction which is stabilized by $\mathcal{P}=\left\{P_{1}, P_{2}\right\}$ where $P_{i} \equiv P_{\mathcal{H}}$ are the projections of $H^{(2)}$ onto its coordinate spaces. Let $G$ be the graph with two vertices $x, y$ and edges $e=y e x, f=x f y$. Then $L_{G}=\left(L_{e}, L_{f}\right)$ in this case. Observe that $C_{2}$ is a deformation (the only one) of $G$. Let $L_{C_{2}}=\left(L_{1}, L_{2}\right)$, where $L_{1}, L_{2}$ are the creation isometries on the full 2 -variable Fock space. Then $L_{e}+L_{f}$ is an isometry and $L_{1}+L_{2}=\sqrt{2} L$ where $L$ is an isometry, and hence

$$
\left\|V_{1}+V_{2}\right\| \leq\left\|L_{e}+L_{f}\right\|=1<\sqrt{2}=\left\|L_{1}+L_{2}\right\| .
$$

Further, the $L_{e}+L_{f}$ estimate is best possible in this example since $\left\|V_{1}+V_{2}\right\|=1$ if $\left\|T_{i}\right\|=1$ for $i=1$ or 2 . 


\section{Functional calculus}

The von Neumann inequality leads to a natural functional calculus determined by quiver algebras and, in the case of pure row contractions, by free semigroupoid algebras. Observe that when $T=\left(T_{e}\right)_{e \in \mathcal{E}(G)}$ is a row contraction with $G$-ordering induced by projections $\mathcal{P}=\left\{P_{x}\right\}$ which satisfy (8) for $T$, we may consider all polynomials $\left\{p(T): p \in \mathcal{P}_{G}^{+}\right\}$in the generators $T_{e}, P_{x}$. The following result is an immediate consequence of Theorem 5.1 and may also be obtained as a special case of Theorem 3.10 from 31.

Theorem 6.1. Let $G$ be a countable directed graph with no sinks. Let $T=$ $\left(T_{e}\right)_{e \in \mathcal{E}(G)}$ be a row contraction on $\mathcal{H}$ which satisfies (8) for $\left\{P_{x}^{\prime}: x \in \mathcal{V}(G)\right\}$. Then there is a contractive homomorphism

$$
\Psi: \mathcal{A}_{G} \longrightarrow \mathcal{B}(\mathcal{H})
$$

defined by $\Psi\left(L_{e}\right)=T_{e}$ and $\Psi\left(P_{x}\right)=P_{x}^{\prime}$ for $e \in \mathcal{E}(G)$ and $x \in \mathcal{V}(G)$.

When the row contraction satisfies an extra condition, the functional calculus can be extended to $\mathfrak{L}_{G}$.

Theorem 6.2. Let $G$ be a countable directed graph with no sinks. Let $T=$ $\left(T_{e}\right)_{e \in \mathcal{E}(G)}$ be a row contraction on $\mathcal{H}$ which satisfies (8) for $\left\{P_{x}^{\prime}: x \in \mathcal{V}(G)\right\}$, and suppose that

$$
\lim _{d \rightarrow \infty}\left(\sum_{w \in \mathbb{F}^{+}(G)_{d}}\left\|w(T)^{*} \xi\right\|\right)=0 \quad \text { for } \quad \xi \in \mathcal{H} .
$$

Then there is a contractive homomorphism

$$
\Psi: \mathfrak{L}_{G} \longrightarrow \mathcal{B}(\mathcal{H})
$$

defined by $\Psi\left(L_{e}\right)=T_{e}$ and $\Psi\left(P_{x}\right)=P_{x}^{\prime}$ for $e \in \mathcal{E}(G)$ and $x \in \mathcal{V}(G)$.

Proof. Condition (11) means precisely that the minimal partially isometric dilation $V=\left(V_{e}\right)_{e \in \mathcal{E}(G)}$ of $T$ with respect to $\left\{P_{x}^{\prime}\right\}$ is pure [19]. By the Wold decomposition for $V$, we may define operators $f(V)$ for all $f \in \mathfrak{L}_{G}$, where $f(V)=$ WOT $-\lim _{k} p_{k}(V)$ for some sequence $p_{k} \in \mathcal{P}_{G}^{+}$. From the properties of the minimal dilation we have $p_{k}(T)=\left.P_{\mathcal{H}} p_{k}(V)\right|_{\mathcal{H}}$. Thus, $p_{k}(T)$ converges to an operator $f(T) \equiv \mathrm{WOT}-\lim _{k} p_{k}(T) \in \mathcal{B}(\mathcal{H})$. Finally, by the von Neumann inequality and a standard approximation argument we have $\|f(T)\| \leq\|f(V)\| \leq\|f(L)\|$, and the result follows.

Remark 6.3. It should be possible to prove an $\mathfrak{L}_{G}$ functional calculus for more general row contractions. For instance, as in [39] 5, 33] this could be accomplished by first identifying "characteristic functions" for an appropriate notion of completely nonunitary row contractions here.

\section{A generalization of Coburn's theorem}

In [8] Coburn showed that the $\mathrm{C}^{*}$-algebra generated by a nonunitary isometry $V$ is isomorphic to the Toeplitz $\mathrm{C}^{*}$-algebra, via a map that sends $V$ to the forward shift. This result was generalized by Popescu to Cuntz-Toeplitz $\mathrm{C}^{*}$-algebras [33]. In this section we consider the generalization of Coburn's Theorem to $\mathrm{C}^{*}$-algebras generated by partial isometries associated with more general directed graphs. 
The proof of Coburn's theorem depends on the universality of the algebra of continuous functions on the circle as the $\mathrm{C}^{*}$-algebra of a graph $G$ consisting of one vertex and one loop-edge. The following result of Szymanski [40] generalizes this fact to arbitrary graph $\mathrm{C}^{*}$-algebras.

Theorem 7.1 (40]). Let $G$ be a countable directed graph with no sources and let $\rho: \mathrm{C}^{*}(G) \longrightarrow \mathcal{A}$ be a*-homomorphism into a $\mathrm{C}^{*}$-algebra $\mathcal{A}$. Then $\rho$ is injective if and only if the following two conditions are satisfied:

(i) $\rho\left(P_{x}\right) \neq 0$ for each vertex $x \in \mathcal{V}(G)$.

(ii) For each vertex simple loop $w \in \mathbb{F}^{+}(G)$ without entrances, we have $\mathbb{T} \subseteq$ $\sigma\left(\rho\left(S_{w}\right)\right)$.

Corollary 7.2. Let $G$ be a countable directed graph with no sources and no sinks. Let $\mathrm{C}^{*}\left(\mathcal{A}_{G}\right)$ be the $\mathrm{C}^{*}$-algebra generated by the quiver algebra $\mathcal{A}_{G}$, let $\mathcal{K}$ denote the set of compact operators on $\mathcal{H}_{G}$ and let $\mathrm{C}^{*}\left(\mathcal{A}_{G}\right) / \mathcal{K}$ denote the image of $\mathrm{C}^{*}\left(\mathcal{A}_{G}\right)$ under the Calkin map. Then there exists a $*$-isomorphism

$$
\rho: \mathrm{C}^{*}(G) \rightarrow \mathrm{C}^{*}\left(\mathcal{A}_{G}\right) / \mathcal{K}
$$

such that $\rho\left(S_{e}\right)=L_{e}+\mathcal{K}$, for all $e \in \mathcal{E}(G)$.

Proof. Since $G$ has no sources, the projections $L_{x}, x \in \mathcal{V}(G)$, are infinite dimensional and thus $L_{x}+\mathcal{K} \neq 0$. Furthermore,

$$
\sum_{r(e)=x} L_{e} L_{e}^{*}=L_{x}-\xi_{x} \otimes \xi_{x}
$$

and so the collections $\left\{L_{x}+\mathcal{K}\right\}_{x \in \mathcal{V}(G)}$ and $\left\{L_{e}+\mathcal{K}\right\}_{e \in \mathcal{E}(G)}$ satisfy the relations $(\ddagger)$. Hence there exists a $*$-homomorphism $\rho: \mathrm{C}^{*}(G) \longrightarrow \mathrm{C}^{*}\left(\mathcal{A}_{G}\right) / \mathcal{K}$ with $\rho\left(P_{x}\right)=$ $L_{x}+\mathcal{K}$ and $\rho\left(S_{e}\right)=L_{e}+\mathcal{K}$ for all $x \in \mathcal{V}(G)$ and $e \in \mathcal{E}(G)$. We now use Szymanski's result to show that $\rho$ is injective.

Towards this end, notice that we have already verified condition (i) in Theorem 7.1 To verify the other condition let $w \in \mathbb{F}^{+}(G)$ be any loop. Consider the direct sum decomposition $\mathcal{H}_{G}=L_{x}\left(\mathcal{H}_{G}\right) \oplus L_{x}\left(\mathcal{H}_{G}\right)^{\perp}$ and notice that with respect to this decomposition, $L_{w}$ admits the $2 \times 2$ matrix form

$$
\left(\begin{array}{ll}
L & 0 \\
0 & 0
\end{array}\right)
$$

with $L$ unitarily equivalent to some ampliation $S^{(\alpha)}$ of the forward shift $S$. It is well known that if $\lambda \in \mathbb{T}$, then the range of $S-\lambda I$ is not closed. Therefore the same is true for $S^{(\alpha)}-\lambda I$ and so $\lambda$ belongs to the essential spectrum of $S^{(\alpha)}$; i.e., $\lambda \in \sigma\left(\rho\left(S_{w}\right)\right)$, as desired.

With a standard "diagram chasing" argument, Corollary 7.2 can be used to show the essential norm coincides with the operator norm for any element of $\mathcal{A}_{G}$. However, we can prove this directly for a larger algebra, the free semigroupoid algebra $\mathfrak{L}_{G}$, hence we include a proof of this fact.

Proposition 7.3. The following assertions are equivalent for a countable directed graph $G$ :

(i) $G$ has no sources.

(ii) $\|A\|=\|A\|_{e}$ for all $A \in \mathfrak{L}_{G}$. 
Proof. If $x$ is a source vertex in $G$, then $P_{x}$ is a rank one projection inside $\mathcal{A}_{G}$ (and $\left.\mathfrak{L}_{G}\right)$. Conversely, suppose $G$ has no sources. Let $d \geq 1$ be a positive integer. For all $x \in \mathcal{V}(G)$ we may choose a path $w_{x} \in \mathbb{F}^{+}(G)$ of length $d$ such that $r\left(w_{x}\right)=x$. As the $w_{x}$ are distinct paths of the same length, notice that the partial isometries $R_{w_{x}}, x \in \mathcal{V}(G)$, have pairwise orthogonal ranges. Thus, we may define an isometry $R^{(d)}=\sum_{x \in \mathcal{V}(G)} R_{w_{x}}$ in the commutant $\mathfrak{L}_{G}^{\prime}=\mathfrak{R}_{G}$ (where the sum converges wot in the infinite vertex case);

$$
\left(R^{(d)}\right)^{*} R^{(d)}=\sum_{x, y} R_{w_{x}}^{*} R_{w_{y}}=\sum_{x} R_{w_{x}}^{*} R_{w_{x}}=\sum_{x} Q_{x}=I .
$$

Hence, if $A$ belongs to $\mathfrak{L}_{G}$, we have

$$
\left\|A R^{(d)} \xi\right\|=\left\|R^{(d)} A \xi\right\|=\|A \xi\| \quad \text { for } \quad \xi \in \mathcal{H}_{G} \quad \text { and } \quad d \geq 1 .
$$

On the other hand, $\left\{R^{(d)} \xi\right\}_{d \geq 1}$ converges weakly to zero for any choice of vector $\xi \in \mathcal{H}_{G}$, and thus $\|A\|=\|A\|_{e}$ as claimed.

Corollary 7.4. If $G$ be a directed graph with no sources, then the compact perturbations $\mathfrak{L}_{G}+\mathcal{K}$ are norm closed.

The next result shows that $\mathrm{C}^{*}\left(\mathcal{A}_{G}\right)$ is the universal $\mathrm{C}^{*}$-algebra for the relations $(\dagger)$ and it is known to the specialists (compare with [32 16]). Nevertheless, we feel that the short proof presented here is new and we therefore include it.

Theorem 7.5. Let $G$ be a countable directed graph with no sources and no sinks. Let $\left\{P_{x}^{\prime}\right\}_{x \in \mathcal{V}(G)}$ and $\left\{S_{e}^{\prime}\right\}_{e \in \mathcal{E}(G)}$ be families of projections and partial isometries acting on a Hilbert space $\mathcal{H}$ which satisfy $(\dagger)$. Then there exists a $*$-epimorphism

$$
\tau: \mathrm{C}^{*}\left(\mathcal{A}_{G}\right) \longrightarrow \mathrm{C}^{*}\left(\left\{S_{e}^{\prime}\right\}_{e \in \mathcal{E}(G)}\right)
$$

such that $\tau\left(L_{e}\right)=S_{e}^{\prime}$, for all $e \in \mathcal{E}(G)$.

Proof. From the discussion in Section 3, it is clear that the Wold decomposition (which was proved for $G$ with no sinks) implies the existence of $\mathrm{C}^{*}$-homomorphisms

$$
\phi_{1}: \mathrm{C}^{*}(G) \longrightarrow B\left(\mathcal{H}_{1}\right)
$$

and

$$
\phi_{2}: \mathrm{C}^{*}\left(\mathcal{A}_{G}\right) \longrightarrow B\left(\mathcal{H}_{2}\right)
$$

such that $\mathcal{H}=\mathcal{H}_{1} \oplus \mathcal{H}_{2}$ and

$$
S_{u}^{\prime} \simeq\left(\phi_{1} \oplus \phi_{2}\right)\left(S_{u} \oplus L_{u}\right) \quad \text { for } \quad u \in \mathbb{F}^{+}(G) .
$$

Consider the diagram

$$
\mathrm{C}^{*}\left(\mathcal{A}_{G}\right) \stackrel{\pi}{\longrightarrow} \mathrm{C}^{*}\left(\mathcal{A}_{G}\right) / \mathcal{K} \stackrel{\rho}{\longrightarrow} \mathrm{C}^{*}(G),
$$

where $\rho$ is the $*$-isomorphism identified by Corollary 7.2. If $\psi \equiv \rho \circ \pi$, then the desired map here is

$$
\tau=\left(\phi_{1} \oplus \phi_{2}\right) \circ(\psi \oplus i d): \mathrm{C}^{*}\left(\mathcal{A}_{G}\right) \longrightarrow \mathrm{C}^{*}\left(\left\{S_{e}^{\prime}\right\}_{e \in \mathcal{E}(G)}\right) .
$$

Tracing the definitions of these maps shows that $\tau\left(L_{u}\right)=S_{u}^{\prime}$ for all $u \in \mathbb{F}^{+}(G)$.

The previous theorem leads to a natural $\mathrm{C}^{*}$-algebra extension of the von Neumann inequality Theorem 5.1 (see also [33] for the case of isometries with orthogonal ranges). Note that the proof of this result relies on Szymanski's Theorem, whereas our proof of Theorem 5.1 is direct. Let $\mathcal{P}_{G}^{0}$ be the set of polynomials generated by 
all indeterminant monomials of the form $v \bar{w}$ with $v, w \in \mathbb{F}^{+}(G)$ and where $x=\bar{x}$ for $x \in \mathcal{V}(G)$. Given $T=\left(T_{e}\right)_{e \in E(G)}$ and $p \in \mathcal{P}_{G}^{0}$, we write $p\left(T, T^{*}\right)$ for the polynomial $p$ evaluated at the generators $T_{e}$ (for $e \in \mathcal{P}_{G}^{0}$ ) and $T_{e}^{*}$ (for $\bar{e} \in \mathcal{P}_{G}^{0}$ ) and $P_{x}$ (for $\left.x=\bar{x} \in \mathcal{P}_{G}^{0} \cap \mathcal{V}(G)\right)$.

Corollary 7.6. Let $G$ be a countable directed graph with no sources and no sinks. Let $T=\left(T_{e}\right)_{e \in E(G)}$ be a row contraction on $\mathcal{H}$ stabilized as in (8) by a given family of projections. Then

$$
\left\|p\left(T, T^{*}\right)\right\| \leq\left\|p\left(L_{G}, L_{G}^{*}\right)\right\| \quad \text { for } \quad p \in \mathcal{P}_{G}^{0} .
$$

Proof. Let $S=\left(S_{e}\right)_{e \in E(G)}$ be the minimal partially isometric dilation of $T$ with respect to the given projection family. From the basic properties of the minimal dilation, it follows that

$$
p\left(T, T^{*}\right)=\left.P_{\mathcal{H}} p\left(S, S^{*}\right)\right|_{\mathcal{H}} \quad \text { for } \quad p \in \mathcal{P}_{G}^{0} .
$$

Thus, Theorem 7.5 gives $\left\|p\left(T, T^{*}\right)\right\| \leq\left\|p\left(S, S^{*}\right)\right\| \leq\left\|p\left(L_{G}, L_{G}^{*}\right)\right\|$ for all $p \in \mathcal{P}_{G}^{0}$.

The next theorem is the promised generalization of Coburn's Theorem. Given a collection $\left\{S_{e}^{\prime}\right\}_{e \in \mathcal{E}(G)}$ of isometries, we isolate a condition which is the appropriate extension of an isometry being nonunitary here (condition (13) below). We show that any collection of partial isometries satisfying this condition generates a $\mathrm{C}^{*}$ algebra isomorphic to $\mathrm{C}^{*}\left(\mathcal{A}_{G}\right)$.

Theorem 7.7. Let $G$ be a countable directed graph with no sources and no sinks and let $\mathrm{C}^{*}\left(\mathcal{A}_{G}\right)$ be the $\mathrm{C}^{*}$-algebra generated by the quiver algebra $\mathcal{A}_{G}$. Let $\left\{P_{x}^{\prime}\right\}_{x \in \mathcal{V}(G)}$ and $\left\{S_{e}^{\prime}\right\}_{e \in \mathcal{E}(G)}$ be families of projections and partial isometries respectively, acting on a Hilbert space $\mathcal{H}$ and satisfying $(\dagger)$. If,

$$
\sum_{r(e)=x} S_{e}^{\prime}\left(S_{e}^{\prime}\right)^{*} \neq P_{x}^{\prime} \quad \text { for } \quad x \in \mathcal{V}(G)
$$

then there exists an injective $*$-homorphism $\phi: \mathrm{C}^{*}\left(\mathcal{A}_{G}\right) \rightarrow B(\mathcal{H})$ such that $\phi\left(L_{e}\right)=$ $S_{e}^{\prime}$ for all $e \in \mathcal{E}(G)$.

In particular, the $\mathrm{C}^{*}$-algebra $\mathrm{C}^{*}\left(\left\{S_{e}^{\prime}\right\}_{e \in \mathcal{E}(G)}\right)$, generated by the collection $\left\{S_{e}^{\prime}\right\}_{e \in \mathcal{E}(G)}$, is isomorphic to $\mathrm{C}^{*}\left(\mathcal{A}_{G}\right)$.

Proof. We utilize the Wold decomposition for the collection $\left\{S_{e}^{\prime}\right\}_{e \in \mathcal{E}(G)}$. Recall the existence of reducing subspaces $\mathcal{H}_{c}$ and $\mathcal{H}_{p}$ for $\left\{S_{e}^{\prime}\right\}_{e \in \mathcal{E}(G)}$ such that $\mathcal{H}=\mathcal{H}_{p} \oplus \mathcal{H}_{c}$ and the restriction of $\left\{S_{e}^{\prime}\right\}_{e \in \mathcal{E}(G)}$ on $\mathcal{H}_{p}$ (respectively $\mathcal{H}_{c}$ ) is pure (respectively fully coisometric). Moreover, if

$$
\mathcal{W} \equiv \operatorname{Ran}\left(I-\sum_{e} S_{e}^{\prime}\left(S_{e}^{\prime}\right)^{*}\right)
$$

then $\mathcal{W}$ is wandering for $\left\{S_{e}^{\prime}\right\}_{e \in \mathcal{E}(G)}$ and the smallest invariant subspace for $\left\{S_{e}^{\prime}\right\}_{e \in \mathcal{E}(G)}$ containing $\mathcal{W}$ equals $\mathcal{H}_{p}$. In addition, the restrictions $\left.S_{e}^{\prime}\right|_{\mathcal{H}_{p}}, e \in \mathcal{E}(G)$, are jointly unitarily equivalent to

$$
\left.\left.S_{e}^{\prime}\right|_{\mathcal{H}_{p}} \simeq \sum_{x \in \mathcal{V}(G)} \oplus L_{e}^{\left(\alpha_{x}\right)}\right|_{\left(Q_{x} \mathcal{H}_{G}\right)^{\left(\alpha_{x}\right)}}
$$


where

$$
\alpha_{x}=\operatorname{rank}\left(P_{x}^{\prime}-\sum_{r(e)=x} S_{e}^{\prime}\left(S_{e}^{\prime}\right)^{*}\right) .
$$

Let $\mathcal{W}_{x}=P_{x}^{\prime} \mathcal{W} \neq 0$ and let $\zeta_{x} \in \mathcal{W}_{x}$ be a unit vector. Notice that the subspaces

$$
\mathcal{M}_{x} \equiv \operatorname{span}\left\{S_{w} \zeta_{x}: w \in \mathbb{F}^{+}(G)\right\}
$$

are reducing for $\left\{S_{e}^{\prime}\right\}_{e \in \mathcal{E}(G)}$ and the restrictions $\left.S_{e}^{\prime}\right|_{M_{x}}, e \in \mathcal{E}(G)$, are jointly unitarily equivalent to $\left.L_{e}\right|_{Q_{x}}, e \in \mathcal{E}(G)$, respectively. Therefore, the subspace $\mathcal{M}=$ $\sum \oplus_{x \in \mathcal{V}(G)} \mathcal{M}_{x}$ is reducing for $\left\{S_{e}^{\prime}\right\}_{e \in \mathcal{E}(G)}$ and the restrictions $\left.S_{e}^{\prime}\right|_{\mathcal{M}_{x}}, e \in \mathcal{E}(G)$, are jointly unitarily equivalent to $L_{e}, e \in \mathcal{E}(G)$. We have therefore established the existence of an epimorphism

$$
\psi: \mathrm{C}^{*}\left(\left\{S_{e}^{\prime}\right\}_{e \in \mathcal{E}(G)}\right) \longrightarrow \mathrm{C}^{*}\left(\mathcal{A}_{G}\right)
$$

mapping generators to generators. It remains to show that $\psi$ is injective.

Consider the diagram

$$
\mathrm{C}^{*}\left(\mathcal{A}_{G}\right) \stackrel{\pi}{\longrightarrow} \mathrm{C}^{*}\left(\mathcal{A}_{G}\right) / \mathcal{K} \stackrel{\rho}{\longrightarrow} \mathrm{C}^{*}(G) \stackrel{\tau}{\longrightarrow} \mathrm{C}^{*}\left(\left\{S_{e}^{\prime} \mid \mathcal{H}_{c}\right\}_{e \in \mathcal{E}(G)}\right),
$$

where $\pi$ is the natural quotient map, $\rho$ is as in Corollary 7.2 and $\tau$ exists because $\mathrm{C}^{*}(G)$ is universal. Let

$$
\phi_{1} \equiv \tau \circ \rho \circ \pi: \mathrm{C}^{*}\left(\mathcal{A}_{G}\right) \longrightarrow \mathrm{C}^{*}\left(\left\{S_{e}^{\prime} \mid \mathcal{H}_{c}\right\}_{e \in \mathcal{E}(G)}\right)
$$

and note that $\phi_{1}\left(L_{e}\right)=\left.S_{e}^{\prime}\right|_{\mathcal{H}_{c}}$, for all $e \in \mathcal{E}(G)$. Consider now the map $\phi$ : $\mathrm{C}^{*}\left(\mathcal{A}_{G}\right) \longrightarrow \mathrm{C}^{*}\left(\left\{S_{e}^{\prime}\right\}_{e \in \mathcal{E}(G)}\right)$, defined as

$$
\left.p\left(L_{e}, L_{e}^{*}\right) \longmapsto p\left(S_{e}^{\prime},\left(S_{e}^{\prime}\right)^{*}\right)\right|_{\mathcal{H}_{p}} \oplus \phi_{1}\left(p\left(L_{e}, L_{e}^{*}\right)\right),
$$

for any noncommutative polynomial $p$. The Wold decomposition shows that $\phi$ is a *-homomorphism and the earlier discussion shows that $\phi\left(L_{e}\right)=S_{e}^{\prime}$, for all $e \in \mathcal{E}(G)$. Therefore, $\phi \circ \psi=i d$ and so $\psi$ is injective, which proves the theorem.

Remark 7.8. It was pointed out to us by the referee that Theorem 7.7 was previously obtained by Fowler and Raeburn [13, Theorem 4.1]. Their approach is quite different from ours since they make an extensive use of Hilbert $\mathrm{C}^{*}$-modules as well as their own methods 14 for analyzing semigroup crossed products. Our proof is shorter and elementary; we only require Szymanski's result, whose proof in [40] is essentially self-contained.

\section{ACKNOWLEDGEMENTS}

The first author was partially supported by a research grant from ECU. The second author was partially supported by an NSERC research grant and start up funds from the University of Guelph. We would like to thank the referee for a number of helpful comments.

\section{NOTE ADDED IN PROOF}

In fact, we have shown that Szymanski's Theorem is not necessary and that one can obtain Theorem [7.7 by using the more elementary "gauge-invariant uniqueness theorem" 21]. 


\section{REFERENCES}

[1] T. Ando, On a pair of commutative contractions, Acta Sci. Math. (Szeged) 24 (1963), 88-90. MR0155193 (27:5132)

[2] W. Arveson, Subalgebras of $\mathrm{C}^{*}$-algebras. III. Multivariable operator theory, Acta Math. 181 (1998), 159-228. MR1668582 (2000e:47013)

[3] C. Badea, G. Cassier, Constrained von Neumann inequalities, Adv. Math. 166 (2002), 260297. MR 1895563 (2003b:47017)

[4] T. Bates, J. Hong, I. Raeburn, W. Szymenski, The ideal structure of the $\mathrm{C}^{*}$-algebras of infinite graphs, Illinois J. Math. 46 (2002), 1159-1176. MR.1988256

[5] J.A. Ball, V. Vinnikov, Functional models for representations of the Cuntz algebra, preprint, 2002.

[6] M. Bożejko, Positive-definite kernels, length functions on groups and a noncommutative von Neumann inequality, Studia Math. 95 (1989), 107-118. MF,1038498 (91f:43011)

[7] J. Bunce, Models for n-tuples of non-commuting operators, J. Funct. Anal. 57 (1984), 21-30. MR 0744917 (85k:47019)

[8] L. Coburn, The $\mathrm{C}^{*}$-algebra of an isometry, Bull. Amer. Math. Soc. 73 (1967), 722-726. MR0213906 (35:4760)

[9] M.J. Crabb, A.M. Davie, von Neumann's inequality for Hilbert space operators, Bull. London Math. Soc. 7 (1975), 49-50. MR0365179 (51:1432)

[10] D. Crocker, A. Kumjian, I. Raeburn, D. Williams, An equivariant Brauer group and actions of groups on $C^{*}$-algebras, J. Funct. Anal. 146, 1997, 151-184. MR,1446378 (98j:46076)

[11] K.R. Davidson, E. Katsoulis, D.R. Pitts, The structure of free semigroup algebras, J. Reine Angew. Math. 533, 2001, 99-125. MR1823866 (2002a:47107)

[12] S.W. Drury, A generalization of von Neumann's inequality to the complex ball, Proc. Amer. Math. Soc. 68 (1978), 300-304. MR0480362 (80c:47010)

[13] N.J. Fowler, I. Raeburn, The Toeplitz algebra of a Hilbert bimodule, Indiana U. Math. J. 48 (1999), 155-181. MR1722197 (2001b:46093)

[14] N.J. Fowler, I. Raeburn, Discrete product systems and twisted crossed products by semigroups, J. Funct. Anal. 155 (1998), 171-204. MR.1623138 (99k:46118)

[15] A. Frazho, Models for non-commuting operators, J. Funct. Anal. 48 (1982), 1-11. MR0671311 (84h:47010)

[16] I. Hirshberg, On the universal property of Pimsner-Toeplitz $\mathrm{C}^{*}$-algebras and their continuous analogues, e-print arxiv math.OA/0310407, preprint, 2003

[17] J.A. Holbrook, Schur norms and the multivariate von Neumann inequality, Recent advances in operator theory and related topics (Szeged, 1999), 375-386, Oper. Theory Adv. Appl., 127, Birkhäuser, Basel, 2001. MF 1902811 (2003e:47016)

[18] F. Jaeck, S.C. Power, Hyper-reflexivity of free semigroupoid algebras, e-print arxiv math.OA/0311201, preprint, 2003.

[19] M.T. Jury, D.W. Kribs, Partially isometric dilations of noncommuting n-tuples of operators, Proc. Amer. Math. Soc. 133 (2005), 213-222. MR2085172 (2005d:47011)

[20] M.T. Jury, D.W. Kribs, Ideal structure in free semigroupoid algebras from directed graphs, J. Operator Theory, to appear.

[21] E. Katsoulis, D.W. Kribs, The $C^{*}$-envelope of the tensor algebra of a directed graph, preprint, 2004.

[22] E. Katsoulis, D.W. Kribs, Isomorphisms of algebras associated with directed graphs, Math. Ann. 330 (2004), 709-728. MR2102309

[23] A. Kumjian, D. Pask $C^{*}$-algebras of directed graphs and group actions, Ergodic Theory Dynam. Systems 19, 1999, 1503-1519. MR 1738948 (2000m:46125)

[24] A. Kumjian, D. Pask, I. Raeburn, Cuntz-Krieger algebras of directed graphs, Pacific J. Math. 184, 1998, 161-174. MR 1626528 (99i:46049)

[25] A. Kumjian, D. Pask, I. Raeburn, J. Renault, Graphs, groupoids, and Cuntz-Krieger algebras, J. Funct. Anal. 144, 1997, 505-541. MR1432596 (98g:46083)

[26] D.W. Kribs, S.C. Power, Free semigroupoid algebras, J. Ramanujan Math. Soc. 19 (2004), 75-117. MR2076898 (2005c:47106)

[27] D.W. Kribs, S.C. Power, Partly free algebras, Proc. International Workshop on Operator Theory and its Applications, Operator Theory: Advances and Applications, BirkhäuserVerlag Basel/Switzerland, 149 (2004), 381-393. 
[28] D. Marshall, Blaschke products generate $H^{\infty}$, Bull. Amer. Math. Soc. 82, 1976, 494-496. MR0402054 (53:5877)

[29] P.S. Muhly, A finite dimensional introduction to operator algebra, A. Katavolos (ed.), Operator Algebras and Application, Kluwer Academic Publishers, 1997, 313-354. MR 1462686 (98h:46062)

[30] P.S. Muhly, B. Solel, Tensor algebras, induced representations, and the Wold decomposition, Can. J. Math. 51 (4), 1999, 850-880. MR.1701345 (2000i:46052)

[31] P.S. Muhly, B. Solel, Tensor algebras over $\mathrm{C}^{*}$-correspondences: representations, dilations, and $\mathrm{C}^{*}$-envelopes, J. Funct. Anal. 158 1998, 389-457. MR1648483 (99j:46066)

[32] M. Pimsner, A class of $\mathrm{C}^{*}$-algebras generalizing both Cuntz-Krieger algebras and crossed products by $\mathbb{Z}$, in "Free Probability Theory" (D. Voiculescu, Ed.), Fields Inst. Comm., vol. 12, 189-212, Amer. Math. Soc., 1997. MR 1426840 (97k:46069)

[33] G. Popescu, Functional calculus for noncommuting operators, Michigan Math. J. 42, 1995, 345-356. MR342494 (96k:47025)

[34] G. Popescu, von Neumann inequality for $\left.(\mathcal{B}(\mathcal{H}))^{n}\right)_{1}$, Math. Scand. 68, 1991, 292-304. MR:1129595 (92k:47073)

[35] G. Popescu, Multi-analytic operators and some factorization theorems, Indiana U. Math. J. 38, 1989, 693-710. MR.1017331 (90k:47019)

[36] G. Popescu, Isometric dilations for infinite sequences of noncommuting operators, Trans. Amer. Math. Soc. 316 (1989), 523-536. MR0972704 (90c:47006)

[37] M. Rosenblum, J. Rovnyak, Hardy classes and operator theory, New York: Oxford University Press, 1985. MR0822228 (87e:47001)

[38] B. Solel, You can see the arrows in a quiver algebra, J. Australian Math. Soc. 77 (2004), 111-122. MF 2069028 (2005d:47115)

[39] B. Sz.-Nagy, C. Foias, Harmonic analysis of operators on Hilbert space, North-Holland, Amsterdam, 1970. MR0275190(43:947)

[40] W. Szymański, General Cuntz-Krieger uniqueness theorem, Internat. J. Math. 13, 2002, 549-555. MR1914564 (2003h:46083)

[41] N.Th. Varopoulos, On an inequality of von Neumann and an application of the metric theory of tensor products to operator theory, J. Funct. Anal. 16 (1974), 83-100. MR0355642 $(50: 8116)$

[42] J. von Neumann, Eine Spectraltheorie fur allgemeine Operatoren eines unitaren Raumes, Math. Nachr. 4 (1951), 258-281. MF.0043386(13:254a)

Department of Mathematics, East Carolina University, Greenville, North Carolina 27858

E-mail address: KatsoulisE@mail.ecu.edu

Department of Mathematics and Statistics, University of Guelph, Guelph, Ontario, CANADA N1G 2W1

E-mail address: dkribs@uoguelph.ca 